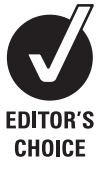

Department of Endocrinology, Sher-i-Kashmir Institute of Medical Sciences, Srinagar, Kashmir, India

Correspondence to: Professor A H Zargar, PO Box 1098, GPO Srinagar 190001. Kashmir, India; zargarah123@ gmail.com

Received 17 January 2008 Accepted 1 October 2008

\title{
Causes of mortality in diabetes mellitus: data from a tertiary teaching hospital in India
}

\author{
A H Zargar, A I Wani, S R Masoodi, M I Bashir, B A Laway, V K Gupta, F A Wani
}

\begin{abstract}
Background: Mortality studies can show the relative contribution of diabetes to mortality in the total population, and they can provide important descriptions of the changes in causes and frequency of diabetes mortality over time.

Objective: To find the mortality pattern in people with diabetes admitted to a tertiary care hospital in a developing country, using underlying/contributory causes of death.
\end{abstract}

Methods: In this retrospective study, mortality trends among people with diabetes admitted to Sher-i-Kashmir Institute of Medical Sciences, Srinagar, Kashmir, India were reviewed by screening the hospital records of all people with diabetes who died over the last 9 years, carrying forward earlier observations from the same centre.

Results: Of 234776 people admitted to the centre during the review period, 16690 (7.11\%) died; 741 (383 men) of these had diabetes mellitus mentioned on the death certificate. The mean (SD) age at death for people with diabetes was 60.07 (13.62) years in men and 57.36 (13.40) years in women. The leading contributory causes of death were infections $(40.9 \%)$, chronic renal failure $(33.6 \%)$, coronary artery disease $(16.9 \%)$, cerebrovascular disease $(13.2 \%)$, chronic obstructive pulmonary disease $(6.9 \%)$, acute renal failure (6.2\%), malignancy $(4.2 \%)$, hypoglycaemia (3.5\%) and diabetic ketoacidosis (3.4\%). The cause of death could not be ascertained in $2.8 \%$ of cases; in $52.9 \%, 36.3 \%$ and $8.0 \%$ cases one, two and three or more causes, respectively, were recorded as the cause of death.

Conclusions: The aetiological spectrum of mortality in people with diabetes at this Indian centre continues to be dominated by infections and renal failure, which is different from that in the developed world, where coronary artery disease and cerebrovascular disease are the principal causes of death in people with diabetes.

The worldwide prevalence of diabetes mellitus (DM) has increased dramatically in the past 20 years. It is one of the most burdensome diseases of our times; between 1985 and 2000, the estimated number of people with DM worldwide increased from 30 to 171 million, ${ }^{12}$ which is projected to rise further to 366 million by the year $2030 .{ }^{3}$ The rising incidence of DM and the sheer number of people with DM living in India has given this developing country the dubious distinction of being the "diabetes capital" of the world. ${ }^{1}$ DM causes significant morbidity and mortality. Even in a developing country such as South Korea, diabetesrelated deaths, which did not figure in the major causes of death in 1983, ranked as the fourth leading cause of mortality following malignancy, cerebrovascular disease and cardiovascular disease (CVD) in 2001. ${ }^{4}$

Obtaining data on the causes of death in people with diabetes is difficult. The difficulty stems from the heterogeneity of diabetes and its misclassification, reliability of reporting of diabetes on death certificates, the reliability and validity of causes of death, and changes in the reporting protocol over time. The bulk of excess risk of mortality among patients with DM in Western countries and Japan has been attributed to circulatory diseases. ${ }^{5-7}$ The scenario is different in Asian and other developing regions of the world where infection and renal failure are more common causes of death among people with diabetes.

About a decade ago, we documented infections, chronic renal failure and coronary artery disease (in that order) as the leading causes of death among 269 people with diabetes who died at our centre between 1987 and $1996 .{ }^{8}$ The mean age at death of diabetic patients in that study was 51.6 years, about 9 years less than the life expectancy of the general population in India at that time. In view of the improved healthcare for people with diabetes over the last decade, we decided to re-examine the mortality trends in our diabetic population. This follow-up work reports on the age at death and causes of mortality among people with diabetes who died at our hospital in the ensuing 9 years, ie, 1997 to 2005. We assume that some deaths occurred at secondary care level or unattended in the community, and the data may represent a selected subgroup of people with diabetes.

\section{MATERIALS AND METHODS}

The methods used in this study are essentially the same as those used in our earlier study. ${ }^{8}$ The data were obtained from the records division of the Institute of Medical Sciences, Soura, Srinagar, Kashmir, India, a 650-bed tertiary care medical centre with most of the modern medical facilities (box 1). There are three levels of healthcare in India: primary, secondary and tertiary. In the Indian context, primary healthcare is provided in the complex of primary health centres by multipurpose workers and village health guides. Secondary level care is provided in district hospitals and community health centres, which serve as the first referral level. The tertiary level, a more specialised level, is provided by regional and central level institutions such as ours. The treatment is mostly free, but this does not affect the referral, as the system is the same at the peripheral level.

Data on the total admissions to Sher-i-Kashmir Institute of Medical Sciences (SKIMS), Srinagar from 1997 to 2005 and the total number of deaths 


\section{Box 1: Study population}

- The Valley of Kashmir in the Northern Indian State of Jammu \& Kashmir has a 2001 census population of 5.5 million.

- The valley has a single major city, Srinagar; the other districts are semi-urban and rural.

- Sher-i-Kashmir Institute of Medical Sciences (SKIMS), Srinagar, a 650-bed hospital, is the only tertiary care medical centre in the Valley of Kashmir.

- Most patients admitted belong to middle or lower socioeconomic groups; treatment is mostly free.

over this period of 9 years were retrieved from the medical records division. After confirmation of death at our centre, the resident-in-charge fills in the death certificate, which is subsequently scrutinised by one of the faculty members of the department concerned. All deaths are reviewed regularly by the "mortality review committee" to authenticate the data. All death certificates that listed DM anywhere in the final diagnosis were retrieved and analysed, especially for the decedent's age, sex, type of diabetes, department to which admitted, duration of hospital stay before death, any complication of diabetes mentioned, any concurrent sickness, and the cause(s) of death. Particular attention was paid to the mention of infection and the type of infection, renal failure, CVD, cerebrovascular disease, diabetic ketoacidosis (DKA), hypoglycaemia and hyperosmolar non-ketotic coma.

\section{Data analysis}

Total number of deaths in diabetic patients during the study period was estimated using the actual number of deaths registered where diabetes was the underlying or a contributory cause. The contributory cause refers to the mention on the death certificate of any cause/disease other than diabetes. When there was mention of only one contributory cause other than diabetes, it was referred to as a single cause. SPSS V11.5 was used for data analysis. Specific statistical tests used were $\chi^{2}$ test for categorical variables, $t$ test for continuous variables, and oneway analysis of variance. All $p$ values were calculated twotailed; $\mathrm{p}<0.05$ was taken as significant.

\section{RESULTS}

A total of 234776 admissions to SKIMS, Srinagar were recorded over a period of 9 years (January 1997 to December 2005). During this period, 16690 (7.1\%) patients died, 741 (4.4\%) of whom had DM recorded on the death certificate. Of the 741 people (383 male) with diabetes who died, constituting the basic material for this study, 41 (5.5\%), $693(93.5 \%)$ and $7(0.9 \%)$ had type $1 \mathrm{DM}$, type $2 \mathrm{DM}$ and fibrocalculous pancreatopathy, respectively. Only $148(20 \%)$ had been admitted to our endocrine unit, and the remaining 593 had been admitted to other departments such as nephrology (32\%), internal medicine $(13 \%)$, neurology $(12 \%)$, cardiology $(10 \%)$, gastroenterology $(5.4 \%)$ and other $(7.3 \%)$.

\section{Age at death}

Figure 1 depicts the age and sex distribution of all 741 subjects in relation to the type of diabetes. The mean (SD) age of death was 58.76 (13.58) (median, 60 years), being significantly lower in women than men 57.4 (13.4) vs 60.1 (13.6) years, respectively; $p=0.007)$. When compared with the average life expectancy at
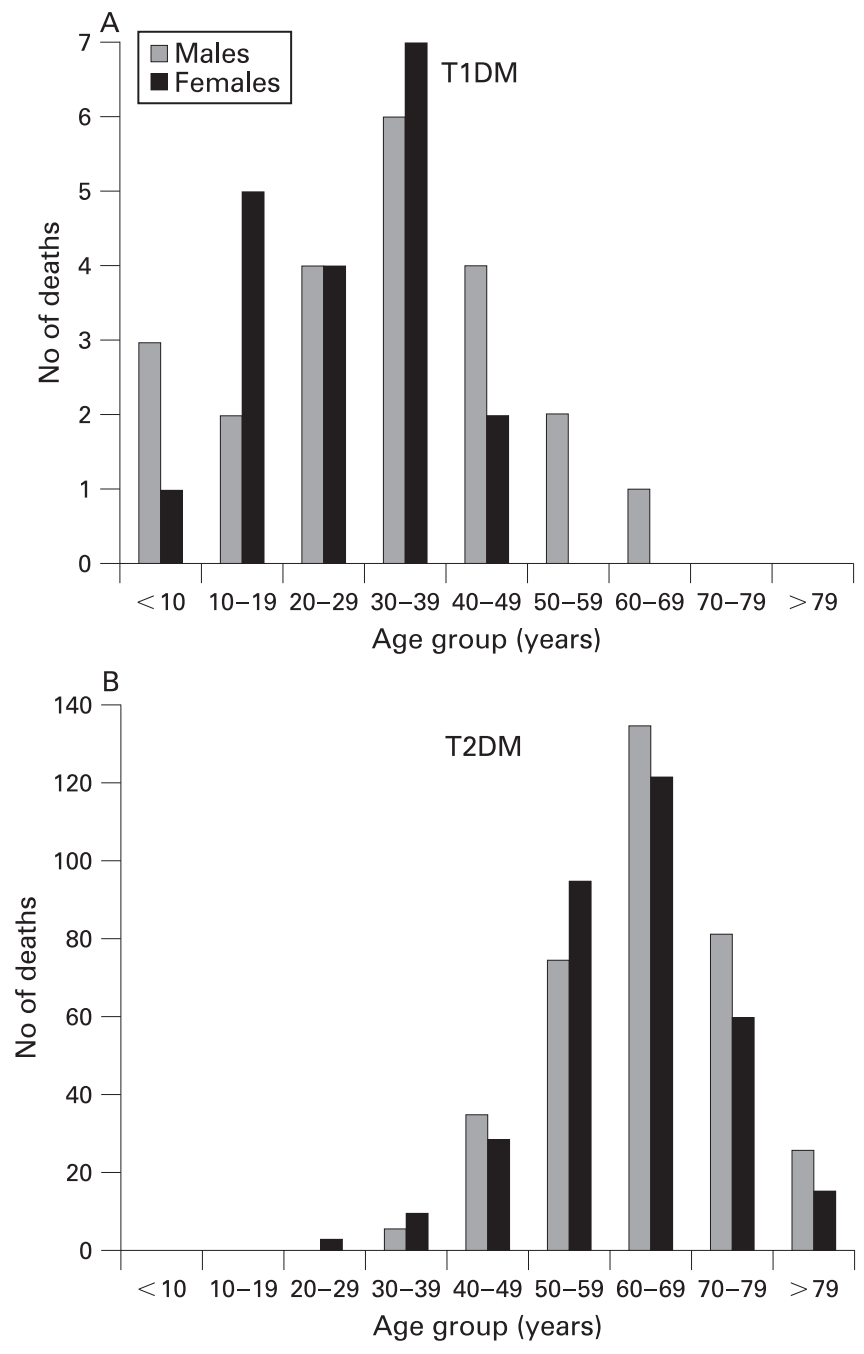

Figure 1 Age and sex distribution of 741 study subjects in relation to type of diabetes. T1DM, type 1 diabetes mellitus; T2DM, type 2 diabetes mellitus.

birth in India (66.9 in women and 63.9 in men; 2001 census), this would mean an average loss of 9.5 years for women compared with 3.8 years for men. As expected, patients with type $2 \mathrm{DM}$ were older than those with type $1 \mathrm{DM}$; mean age at death was 29.61 (13.34), 60.66 (11.34) and 41.71 (12.41) years, respectively, in people with type1 DM, type 2 DM and fibrocalculous pancreatopathy. Female patients with type 1 DM died youngest, with a mean age at death of 27.26 (11.06) years.

\section{Causes of death}

The leading causes to which death was attributed in these 741 people with diabetes included infections (40.9\%), chronic renal failure $(33.6 \%)$, coronary artery disease $(16.9 \%)$ and cerebrovascular disease (13.2\%) (table 1). Among the acute metabolic complications of diabetes, hypoglycaemia, DKA and hyperglycaemic hyperosmolar state were attributed as the cause of death in $26(3.5 \%), 25(3.4 \%)$ and $17(2.3 \%)$ patients, respectively. In $21(2.8 \%)$ patients, the records were not clear about the cause of death. In patients with type 1 DM, DKA was the second most common cause of death after infections, accounting for 17 $(41.5 \%)$ deaths (table 2$)$. No pregnancy-related deaths were noted in the hospital records. 
Table 1 Causes of death in 741 patients with diabetes

\begin{tabular}{lcc}
\hline & Contributory cause & Single cause \\
\hline Infections & $303(40.9)$ & $94(12.7)$ \\
Chronic renal failure & $249(33.6)$ & $105(14.2)$ \\
Coronary artery disease & $125(16.9)$ & $52(7.0)$ \\
Cerebral vascular accident & $98(13.2)$ & $50(6.7)$ \\
Chronic obstructive pulmonary disease & $51(6.9)$ & $12(1.6)$ \\
Acute renal failure & $46(6.2)$ & $8(1.1)$ \\
Malignancy & $31(4.2)$ & $11(1.5)$ \\
Chronic liver disease & $20(2.7)$ & $9(1.2)$ \\
Hypoglycaemia & $26(3.5)$ & $6(0.8)$ \\
Cardiomyopathy/heart blocks & $24(3.2)$ & $6(0.8)$ \\
Diabetic ketoacidosis & $25(3.4)$ & $5(0.7)$ \\
Hyperglycaemic hyperosmolar state & $17(2.3)$ & $5(0.7)$ \\
Gastrointestinal bleed & $18(2.4)$ & $4(0.5)$ \\
Seizures & $13(1.8)$ & $4(0.5)$ \\
Acute respiratory distress syndrome & $11(1.5)$ & $4(0.5)$ \\
Pulmonary thromboembolism & $11(1.5)$ & $3(0.3)$ \\
Acute pancreatitis & $6(0.8)$ & $2(0.3)$ \\
Road traffic crash & $5(0.7)$ & $2(0.3)$ \\
Other miscellaneous & $32(4.3)$ & $10(1.3)$ \\
Multiple causes & - & $328(44.3)$ \\
Undetermined cause & - & $21(2.8)$ \\
\hline
\end{tabular}

Values in parentheses are percentages. Contributory cause refers to the mention of any cause on the death certificate, whereas single cause refers to the only cause mentioned other than diabetes.

Death was attributed to a single cause in $392(52.9 \%)$, two causes in $269(36.3 \%)$, and three or more causes in $59(8.0 \%)$ cases. While infection in combination with other causes was the most common cause of death in our patients with diabetes, as a single cause, chronic renal failure was the most common (table 1). There was no significant difference in the age and mortality pattern among people with one or more causes of death except in the chronic renal failure group. The mean age at death was lower when chronic renal failure was the single cause of death than when it was a contributory cause in people for whom three or more causes of death were noted (58.8 (11.4) vs $65.6(12.2)$ years, respectively; $p=0.02$ ).

\section{Cause of death in the young}

About a sixth $(127,17.1 \%)$ of all deaths occurred in people with diabetes of $<50$ years of age: $53(7.2 \%)$ in the young ( $<40$ years) and $74(10 \%)$ in early middle age (40-49 years). The most common causes of death, in addition to diabetes, in the young $(<40$ years) included infections in $31(58.5 \%)$, DKA in 16 (30.2\%), chronic renal failure in $12(22.6 \%)$, and hypoglycaemia in five (9.4\%); however, 28 (52.8\%) had multiple causes. Among patients $40-49$ years old $(n=74)$, chronic renal failure was the most common cause of death (36.5\%) and the most common single cause of death (17.6\%).

\section{Cause of death in older people}

More than half $(442,59.6 \%)$ of all deceased people with diabetes were 60 years or older, and about a quarter $(183,24.7 \%)$ were aged 70 years or more. Compared with this, only $40 \%$ were 60 years or older and just $10 \%$ were 70 years or older in our earlier study. ${ }^{8}$

\section{Gender versus mortality pattern}

Apart from younger age at death, the only other factors that were significantly different in women were higher frequency of DKA $(4.7 \%$ vs $2.1 \%$, respectively; $p=0.045)$ and lower
Table 2 Causes of death in 741 patients with diabetes mellitus in relation to type of diabetes

\begin{tabular}{lccl}
\hline & $\begin{array}{l}\text { Type 1 } \\
(\mathbf{n}=\mathbf{4 1})\end{array}$ & $\begin{array}{l}\text { Type 2 } \\
(\mathbf{n}=\mathbf{6 9 3})\end{array}$ & p Value \\
\hline Infections & $21(51.2)$ & $280(40.4)$ & 0.2 \\
Chronic renal failure & $7(17.1)$ & $239(34.5)$ & 0.02 \\
Coronary artery disease & 0 & $125(18.0)$ & 0.001 \\
Cerebral vascular accident & $5(12.2)$ & $93(13.4)$ & 0.8 \\
Chronic obstructive pulmonary disease & 0 & $51(7.4)$ & 0.1 \\
Acute renal failure & $2(4.9)$ & $43(6.2)$ & 0.7 \\
Malignancy & $2(4.9)$ & $29(4.2)$ & 0.8 \\
Hypoglycaemia & $4(9.8)$ & $22(3.2)$ & 0.049 \\
Diabetic ketoacidosis & $17(41.5)$ & $8(1.2)$ & 0.000 \\
Cardiomyopathy/heart blocks & 0 & $24(3.5)$ & 0.4 \\
Chronic liver disease & $1(2.4)$ & $18(2.6)$ & 0.9 \\
Road traffic crash & $1(2.4)$ & $4(0.6)$ & 0.3 \\
Seizures & 0 & $12(1.7)$ & 0.4 \\
Hyperglycaemic hyperosmolar state & $1(2.4)$ & $16(2.3)$ & 0.9 \\
Acute pancreatitis & 0 & $5(0.7)$ & 0.9 \\
Acute respiratory distress syndrome & $2(4.9)$ & $9(1.3)$ & 0.1 \\
Gastrointestinal bleed & 0 & $18(2.6)$ & 0.5 \\
Pulmonary thromboembolism & 0 & $11(1.6)$ & 0.5 \\
Other miscellaneous & $3(7.3)$ & $29(4.2)$ & 0.4 \\
\hline Values in paran & &
\end{tabular}

Values in parentheses are percentages.

frequency of microvascular complications $(11.7 \%$ vs $19.6 \%$, respectively; $p=0.03$ ).

Rural habitat versus mortality pattern

A rural background was noted in $41 \%$ of cases. Some differences in mortality pattern were observed in subjects of rural and urban origin (table 3).

\section{Hospital stay}

Overall, the hospital stay ranged from 1 to 50 days with a mean (SEM) of 4.9 (6.4) days (median, 2). The mean (SEM) stay was 7.51 (1.66), $4.70(0.23)$ and 6.43 (2.09) days for patients with type $1 \mathrm{DM}$, type $2 \mathrm{DM}$ and fibrocalculous pancreatopathy, respectively $(p<0.02)$. Patients for whom coronary artery disease was a contributory cause of death had a shorter hospital stay than those for whom it was not $(3.32$ (3.87) vs 5.19 (6.71) days; $p=0.003$ ).

\section{DISCUSSION}

This is the largest hospital-based study of mortality pattern in patients with diabetes from North India and presents two main findings: (1) the mean age at death in hospital is significantly lower than the life expectancy at birth in India; (2) infections and renal disease contribute to most deaths of people with

Table 3 Age at death and causes that were significantly different in rural and urban populations

\begin{tabular}{lccl}
\hline Variable & $\begin{array}{l}\text { Urban } \\
(\mathbf{n}=\mathbf{4 4 0})\end{array}$ & $\begin{array}{l}\text { Rural } \\
(\mathbf{n}=\mathbf{3 0 1})\end{array}$ & $\mathbf{p ~ V a l u e}$ \\
\hline Age (years) & $60.3(11.9)$ & $56.5(15.5)$ & 0.000 \\
Type 1 diabetes mellitus (\%) & 2.7 & 9.6 & 0.000 \\
Sepsis (\%) & 10.0 & 16.6 & 0.006 \\
Chronic renal failure (\%) & 38.0 & 27.2 & 0.002 \\
Coronary artery disease (\%) & 19.3 & 13.3 & 0.031 \\
Diabetic ketoacidosis (\%) & 2.0 & 5.3 & 0.015 \\
Seizures (\%) & 0.9 & 3.0 & 0.034 \\
Pulmonary thromboembolism (\%) & 0.7 & 2.7 & 0.029 \\
\hline
\end{tabular}

*Mean (SD). 
Current research questions

- Is there any change in mortality pattern over the last decade in comparison with previous data from the same tertiary-care centre in North India?

- Is there a diminishing trend for infection to be the number one cause of death in people with diabetes admitted to the tertiary centre?

diabetes in this part of the world. As life expectancy in patients with diabetes is difficult to predict because of many confounding variables, we believe that evaluation of data from death certificates is a relatively simple way of determining the mortality pattern.

We found that between 1997 and 2005, the mean age of death in hospital (60.1 years in men and 57.4 years in women with diabetes) was significantly lower than the average life expectancy (63.9 years in men; 66.9 years in women) in India. Moreover, $17 \%$ of our patients had died at an age of $<50$ years. Comparing this with our previous study (1987-1996 period) in which 9267 patients of 133374 admissions (7\%) had died, 269 (2.8\%) were recorded to have $\mathrm{DM} .^{8}$ The mean age at death of patients with diabetes in that study was 51.6 years, about 9 years lower than the life expectancy of the general population in India at that time. ${ }^{8}$ Although these figures represent an improvement from earlier figures, the life span of a patient with diabetes continues to be shortened in our community, especially for women, who died on average 9.5 years earlier than their life expectancy at birth. In addition, we do not know what would have been the life expectancy of our population at the time of diagnosis of diabetes, although it would have been definitely better than that at birth. Therefore, diabetes exacts a heavy toll in terms of years of productive life lost, which undoubtedly will be much greater than suggested by the mean age at demise of our patients with diabetes.

The worst scenario was observed in women with type $1 \mathrm{DM}$ who died at an average age of 27 years. Whether this reflects a bias in the community towards healthcare of its female folk, the tolerant and less complaining attitude of women in this part of the world, the lack of health awareness in women, or an incidental observation is beyond the scope of this study. Although our hospital death rate has remained almost the same, the proportion of death certificates mentioning diabetes has increased by about $60 \%$ since the previous study. ${ }^{8}$ This increase in the proportion of deaths from diabetes probably reflects an increase in the incidence of diabetes with the changing criteria for diagnosis and improved death certificate data. However, it is impossible to identify the relative contribution of these factors to the increased proportion of death certificates mentioning diabetes over time.

Studies from the West and the more developed Asian regions report the highest death rate among people with diabetes in the age group $\geqslant 75$ years. ${ }^{9-11}$ In our study, only one-quarter of people with diabetes died at the age of $\geqslant 70$. Very little information is available from India on the proportion of death certificates for people with diabetes that mention diabetes; an Indian study by Das et $a l^{12}$ reported the age at the time of death for people with diabetes to be 55-61 years. In this study, which was carried out over 1 year, it was observed that mortality among hospitalised patients with non-insulin-dependent DM was nearly $20 \%$. The mean age at death was 61 years in those who died within 1 month, ${ }^{12}$ which is almost same as that observed in our study

\section{Main messages}

- The average age at death for people with diabetes (men, 60 years; women, 57 years) has improved compared with previous data but continues to be lower than the average life expectancy at birth in India.

- The aetiological spectrum of mortality in people with diabetes at our centre continues to be dominated by infections $(41 \%)$ and renal failure $(32 \%)$, which is different from that in the developed world where coronary artery disease and cerebrovascular disease are the principal causes of death in people with diabetes.

carried out two decades later. One can argue that our study, as well as that of Das et al, ${ }^{12}$ is hospital based, and hence the findings cannot be extrapolated to a community where many deaths occur unattended. A recent population-based study from South India by Mohan et a ${ }^{13}$ reported the mean age of death among people with diabetes to be 66 years, with death rates twice as high as in non-diabetic subjects. However, populationbased studies also have their limitations, such as no hard evidence of the cause of death, inaccuracy of verbal accounts, non-availability of medical records and small numbers, as pointed out by the authors of this study. ${ }^{13}$

Seven percent of all deaths in our study occurred in patients younger than 40 years, most of whom had type 1 DM. Although this is lower than the previous figure of $15 \%,{ }^{8}$ it continues to be disconcerting. It probably reflects a mixture of improved survival in younger people with diabetes and a higher number of older people having DM diagnosed and recorded on their death certificates. In the modern era of insulin and insulin analogues, very few young patients should, or do, succumb to acute metabolic complications of diabetes; however, in the underdeveloped areas of the world such as India, they continue to be susceptible to major morbidity and early demise.

Infections, alone or in combination with other causes, accounted for more than $40 \%$ of deaths in our study. Earlier studies found infections to be a significant cause of mortality even in developed countries. ${ }^{8} 14{ }^{15}$ However, more recent studies from developed and some developing countries found that a much smaller proportion of death certificates for people with diabetes mentioned infectious disease. ${ }^{1016}{ }^{17}$ Similarly, in the South Indian study, ${ }^{13}$ death due to infection was found to be very low $(\sim 6 \%)$. As ours was a hospital-based study, the prevalence of infection may have been higher. However, the low proportion of infections in the South Indian study may also be a biased estimate because of a lower frequency of infections overall in the community studied, the small sample size, possible dilution of infection cases by nearby hospitals, and other problems that are inherent to population-based studies. Nonetheless, the continued dominance of infections in causing mortality in our patients with diabetes may stem from the overall high incidence of infections in this part of the world, deriving from low literacy rates and poor public health infrastructure, and also the poor control of glycaemia in people with diabetes. ${ }^{18}$

Chronic renal failure was the second most common cause of death in our study, accounting for about one-third of deaths. It was also the most common "single cause" of death (15\%). This reflects the generally poor glucose and hypertension control in these patients with diabetes. Renal failure was more often observed in patients from an urban background. However, it 
was beyond the scope of this study to determine whether the higher proportion of deaths from renal failure in urban than rural areas reflects migration to the city for treatment. Although renal replacement therapy (dialysis/transplant) is available for patients with end stage renal disease at our centre, the demand is much greater than our centre can satisfy. We hope that, with greater availability of such treatment and improvement in socioeconomic status, mortality from renal disease will be reduced. However, renal failure continues to be an important cause of mortality in people with diabetes, especially in underdeveloped countries such as India. In the populationbased study by Mohan et al, ${ }^{13}$ the second most common cause of death in patients with diabetes was renal disease, accounting for $23.5 \%$ of deaths, which is similar to figures reported in other studies. Also, the possibility of genetic susceptibility to kidney disease, as has been suggested in some races, ${ }^{19}$ cannot be excluded in our population.

CVDs including diseases of the cerebral circulation are by far the most common cause of death in patients with DM, accounting for more than half. ${ }^{10} 162021$ However, these findings are mainly from studies in more developed countries. In the recent South Indian study, CVD was also the most common aetiology, accounting for mortality in nearly $53 \%$ with a hazard ratio of 3.5. In our study, these disorders were the third major cause of mortality (30.09\%), some way behind infections and renal disease. It is well known that the rate of reporting of diabetes on CVD death certificates remains poor. ${ }^{22}$ This may indicate a lack of awareness of the importance of diabetes as a risk factor for CVD. Other reasons for the lower contribution of CVD to diabetic mortality in our study may be the demise of some young patients from infections before they could die from CVD, and, also because many CVD-related deaths may occur before patients make it to the hospital because of difficult terrain, poor transport and frequent disturbances due to the prevailing political turmoil over the last 20 years. The contribution of CVD to mortality in our population is also likely to increase if and when there is more availability of renal replacement therapy for our patients with renal failure.

Complications related to DKA are the most common cause of death in children, teenagers and young adults with diabetes; DKA causes up to a third of all deaths in people with diabetes younger than 24 years. ${ }^{23}$ Compared with this, DKA was the cause of death in $63 \%$ of our patients with diabetes who were younger than 24 years; overall, it was a direct or contributory cause of death in about $3 \%$ of our patients with diabetes. Although it reflects an improvement in mortality from DKA in our population over previous figures of $7 \%,{ }^{8}$ it is still higher than the $1.3 \%$ reported for our neighbouring country of China. ${ }^{24}$ Hypoglycaemia, which is largely preventable, is not an uncommon cause of mortality or major morbidity requiring hospitalisation. In some earlier studies, it was considered to be the primary or contributing cause of death in up to $4 \%$ of patients with diabetes. ${ }^{25}$ Our results showing that $3.5 \%$ of all deaths in people with diabetes were caused directly, or partly, by hypoglycaemia attest to this observation.

The other notable causes of death in people with diabetes in our study include chronic respiratory disorders $(6.9 \%)$, acute renal failure $(6.2 \%)$, malignancy $(4.2 \%)$, cardiac disorders other than coronary artery disease (3.2\%), liver disease $(2.7 \%)$, gastrointestinal bleeding $(2.4 \%)$, adult respiratory distress syndrome $(1.5 \%)$ and pulmonary thromboembolism $(1.5 \%)$. The proportion of these causes of death, seemingly unrelated to DM, in our study is different from that reported in an earlier study from China. ${ }^{24}$ In this study by Wei et al, 1383 death certificates filed between 1987 and 1990 at the Kaohsiung Medical College Hospital were reviewed to investigate causes of death in 151 patients with diabetes (infection (25.8\%), CVD $(18.5 \%$, ) cerebrovascular disease $(11.3 \%)$, uraemia $(8.6 \%)$ and DKA $(1.3 \%)$ ), with diabetes reported as the contributory or underlying cause of death. ${ }^{24}$

Another finding of our study was that there were some differences in patients from rural and urban backgrounds, with patients of rural origin dying at a younger age, being more likely to have type 1 diabetes and infections, and being less likely to have renal disease or CVD.

In conclusion, we have found that infective disorders and renal failure continue to play a dominant role in diabetes-related mortality among people admitted to a tertiary care hospital in this part of the world, in contrast with the developed world where coronary artery disease and cerebrovascular diseases dominate. There is a pressing need to address the deficiencies in healthcare of patients with diabetes and associated co-morbidities such as hypertension to lessen the burden of morbidity and premature mortality in this ever increasing patient population. Comprehensive, and quick, measures need to be taken to lessen and eliminate the avoidable mortality from infections. Reducing the risk of infection by strict glycaemic control, early referral, aggressive treatment of infections in hospital, and creating awareness about preventive strategies for microvascular and macrovascular complications of diabetes may help in reducing diabetic mortality in our patients.

Acknowledgements: We are grateful to the staff of the Medical Records Division of Sher-i-Kashmir Institute of Medical Sciences (SKIMS), Srinagar, Kashmir, especially Mohammad Akram, Mohammad Yousuf Dar, Ghulam Ahmad Shah and Farooq Ahmad Gilkar for their help in data collection.

\section{Competing interests: None.}

Ethics approval: Obtained.

\section{REFERENCES}

1. King H, Aubert RE, Herman WH. Global burden of diabetes, 1995-2025: prevalence, numerical estimates, and projections. Diabetes Care 1998;21:1414-31.

2. Wild S, Roglic G, Green A, et al. Global prevalence of diabetes: estimates for the year 2000 and projections for 2030. Diabetes Care 2004;27:1047-53.

3. World Health Organization. Global strategy on diet, physical activity, and health: diabetes. 1 Jan 2006. http://www.who.int/dietphysicalactivity/publications/facts/ diabetes/en/index.html (accessed 6 Apr 2009).

4. National Statistical Office. Yearbook of causes of death statistics in Korea. Seoul: Government Printing, 2001

5. Muhammad U, Ali M, Panneerselvam A. Mortality in diabetes. International Journal of Diabetes in Developing Countries 1994;14:113-15.

6. Simons LA, McCallum J, Friedlander $Y$, et al. Diabetes mellitus and coronary artery disease in the prospective Dubbo Study of Australian elderly. Aust N Z J Med 1996;26:66-74.

7. Michealis D, Jutzi E. Trends in mortality rates in the diabetic population of the GDR Exp Clin Endocrinol 1990;95:83-90.

8. Zargar AH, Wani Al, Masoodi SR, et al. Mortality in diabetes mellitus: data from a developing region of the world. Diabetes Res Clin Pract 1999;43:67-74.

9. Balkau B, Papoz L. Certification of cause of death in French diabetic patients J Epidemiol Community Health 1992;46:63-5.

10. de-Marco R, Locatelli F, Zoppini G, et al. Cause-specific mortality in type 2 diabetes The Verona Diabetes Study. Diabetes Care 1999;22:756-61.

11. Lipscombe LL, Hux JE. Trends in diabetes prevalence, incidence, and mortality in Ontario, Canada 1995-2005: a population-based study. Lancet 2007;369:750-6.

12. Das S, Mishra RK, Jena BB, et al. Mortality events amongst non-insulin diabetes mellitus patients in Orissa. Journal of the Association of Physicians India 1991:39:519-20.

13. Mohan V, Shanthirani CS, Deepa M, et al. Mortality rates due to diabetes in a selected urban south Indian population: the Chennai Urban Population Study (CUPS 16). Journal of the Association of Physicians India 2006;54:113-17.

14. Bisi H, Ruggeri GB, Longatto-Filho A, et al. Study in necropsy material of 'cause specific mortality' in diabetics in Sao Paulo, Brazil. Rev Paul Med 1993;111: 229-304

15. Ito H, Araki A. Course, prognosis and mortality in Japanese elderly diabetes mellitus: a 7-year follow up study. Nippon Ronen Igakkai Zaashi 1993;30:277-82. 
16. Tseng CH. Mortality and causes of death in a national sample of diabetic patients in Taiwan. Diabetes Care 2004;27:1605-9.

17. McEwen LN, Kim C, Haan M, et al. Diabetes reporting as a cause of death: results from the Translating Research Into Action for Diabetes (TRIAD) study. Diabetes Care 2006;29:247-53

18. Ahuja MMS. Causes of death amongst diabetics. International Journal of Diabetes in Developing Countries 1994;14:111-12.

19. Espino DV, Parra EO, Kriehbiel R. Mortality differences between elderly Mexican Americans and non-Hispanic whites in San Antanio, Texas. J Am Geriatr Soc 1994; 42:604-8.

20. Morgan CL, Currie CJ, Peters JR. Relationship between diabetes and mortality: a population study using record linkage. Diabetes Care 2000;23:1103-7.
21. Choi YJ, Cho YM, Park CK, et al. Rapidly increasing diabetes-related mortality with socio-environmental changes in South Korea during the last two decades. Diabetes Res Clin Pract 2006;74:295-300.

22. Thomason MJ, Biddulph JP, Cull CA, et al. Reporting of diabetes on death certificates using data from the UK Prospective Diabetes Study. Diabet Med 2005;22:1031-6.

23. White NH. Diabetic ketoacidosis in children. Endocrinol Metab Clin North Am 2000;29:657-82.

24. Wei SY, Shin SJ, Chen JH, et al. Major causes of diabetic death at one hospital. Kao-Hsiung-I-Hsueh-Ko-Hsuch-Tsa-Chin 1996;12:216-20.

25. Turnbridge WMG. Factors contributing to death of diabetics under fifty years of age. Lancet 1981;2:569-72. 